\title{
Transatlantica
}

Revue d'études américaines. American Studies Journal

$1 \mid 2002$

Jeune République

\section{Les créoles louisianais défendent la cause du Sud à Paris (1861-1865)}

Latinisme contre anglosaxonisme

Salwa Nacouzi

\section{CpenEdition}

Journals

Édition électronique

URL : http://journals.openedition.org/transatlantica/451

DOI : $10.4000 /$ transatlantica.451

ISSN : $1765-2766$

Éditeur

AFEA

Référence électronique

Salwa Nacouzi, «Les créoles louisianais défendent la cause du Sud à Paris (1861-1865) »,

Transatlantica [En ligne], 1 | 2002, mis en ligne le 23 mars 2006, consulté le 29 avril 2021. URL : http://

journals.openedition.org/transatlantica/451 ; DOI : https://doi.org/10.4000/transatlantica.451

Ce document a été généré automatiquement le 29 avril 2021.

\section{c) (1) $(9)$}

Transatlantica - Revue d'études américaines est mis à disposition selon les termes de la licence Creative Commons Attribution - Pas d'Utilisation Commerciale - Pas de Modification 4.0 International. 


\title{
Les créoles louisianais défendent la cause du Sud à Paris (1861-1865)
}

Latinisme contre anglosaxonisme

\author{
Salwa Nacouzi
}

1 La guerre de Sécession a fait l'objet d'analyses exhaustives et les arguments des Sudistes pour le maintien de l'esclavage ont été largement étudiés. En revanche, les campagnes de propagande menées par les représentants du gouvernement des Confédérés auprès des puissances européennes n'ont que peu retenu l'attention et les rares publications sur ce sujet s'attachent essentiellement aux activités menées par les responsables officiellement désignés.

2 L'originalité des actions menées en France par les créoles ${ }^{1}$ n'a jamais vraiment fait l'objet d'une analyse spécifique, or les Louisianais et surtout les créoles, vont s'investir pleinement dans la défense de leur cause, à Paris, où ils séjournent pendant la guerre. Leur combat perdu d'avance mérite qu'on s'y attarde, car il permet, d'une part, d'éclairer la nature des liens qui les unissent encore à la mère-patrie, les espoirs qu'ils y fondent et le désaveu qu'ils rencontrent et, d'autre part, d'étudier la spécificité de la double appartenance de cette communauté.

3 En 1860, les Louisianais gardent encore des liens très étroits avec la France et ils continuent d'y envoyer leurs enfants afin qu'ils y poursuivent des études au lycée Louis le Grand puis à la Faculté de droit ou de médecine. La Nouvelle-Orléans, malgré son architecture espagnole et son administration américaine, demeure une ville de culture française (http ://www.lib.lsu.edu/special/exhibits/creole), ses écrivains rêvent de se faire éditer en France, ses journaux rapportent fidèlement les événements politiques et culturels de la mère patrie ${ }^{2}$. La France est ainsi le lieu de villégiature de «l'aristocratie » créole : on va prendre les eaux à Vichy, puis on se rend à l'opéra de Paris pour y assister à une représentation.

4 Lorsqu'éclatent les hostilités, ces Louisianais se sentent les tenants légitimes d'une mission : celle de défendre auprès de leurs « compatriotes » leurs terres et leur mode de vie. Lorsqu'ils se rendent compte que la France a changé, que l'opinion publique est très hostile à l'esclavage aboli depuis 1848 , ils élaborent des stratégies pour rallier le monde 
politique et l'opinion publique à leur cause. Alors qu'en Angleterre la propagande menée par Henry Hotze joue sur le passé commun anglo-saxon et protestant, les créoles, eux, vont mettre en exergue leur héritage latin commun. Cette approche très opportuniste s'explique par la situation politique en France. En effet, les Sudistes avaient contre eux à la fois, les monarchistes français orléanistes et bourbons hostiles à l'Empire et les républicains défenseurs de l'abolition; leur seul espoir résidait donc dans les ambitions de Napoléon III au Mexique. C'est en pleine guerre de Sécession que l'Empereur installe l'archiduc Maximilien à la tête du Mexique. Il rêve sans doute d'une puissante nation latine et catholique qui ferait contrepoids à la nation anglo-saxonne et protestante des États-Unis. Les créoles louisianais vont mettre l'accent sur la nécessité d'une telle nation pour essayer de tirer Napoléon III de sa neutralités.

5 À travers le parcours et les écrits parisiens, essentiellement des pamphlets (http :// www.lib.lsu.edu/special/), de trois hommes créoles issus des grandes plantations esclavagistes et francophones ${ }^{4}$ pendant les années de guerre, je me propose, dans cet article, de dégager une typologie de ce groupe, convaincu de la légitimité de sa cause et jouant, pour la défendre, de sa double appartenance au Sud et à la France. ${ }^{5}$

1. Paul Pecquet Du Bellet Du Verton

6 Le choix de Paul Pecquet du Bellet du Verton s'impose car, lorsque la guerre de Sécession éclate, du Bellet, ancien attorney-at-law à la Nouvelle-Orléans vit à Paris depuis déjà six ans et sera le premier à lancer la propagande sudiste dans les journaux français afin de contrer le représentant du gouvernement de Washington à Paris, John Biglow.

7 Jusqu'en 1998, nous n'avions que peu d'informations sur sa vie. Stanley Hoole qui édite, en 1963, son "Memorandum " ${ }^{6}$ déclare n'avoir rien trouvé sur le lieu précis de sa naissance, alors que du Bellet lui-même, dans son manuscrit, affirme être né à La Nouvelle-Orléans. On trouve dans les registres de la ville (New Orleans Directory and Registry de 1827) son nom et son lieu de résidence (Royal Street), mais, à partir de 1855, on perd sa trace. Il vivait probablement déjà à Paris car, en 1871, il affirme y résider depuis 15 ans. En mars 1998, Nicolas Kariouk offre à la Hill Memorial Library de la Louisiana State University des documents rassemblés par son trisaïeul Pecquet du Bellet de Verton ${ }^{7}$. Dans ces documents on retrouve effectivement les dates de naissance et de décès (Nouvelle-Orléans, le 6 avril 1816 / Paris, le 21 janvier 1884) qui corroborent ses origines créoles. Par ailleurs, d'après Noémie Pecquet, fille de Paul, qui tente de reconstituer l'arbre généalogique familial, le premier Pecquet quitte la France en 1793, s'installe à Saint-Domingue puis, après la Révolution française, se réfugie en Louisiane. Là, il épouse une veuve et s'installe dans une plantation où naîtront ses deux enfants, dont Paul.

Défenseur acharné des States' rights, du Bellet se rallie immédiatement à la cause des Confédérés et, bien que parfois critique envers la politique du Président Jefferson Davis et de son gouvernement, il se montre très actif dans la défense du Sud, à Paris. Très rapidement, il collabore avec d'autres sympathisants du Sud, en particulier avec Edwin de Leon, ami personnel du président Jefferson Davis, envoyé en avril 1862 pour organiser la propagande confédérée en France-à l'instar d'Henry Hotze en Angleterre. La principale contribution de de Leon, outre le versement de pots de vin aux journalistes français, sera la publication d'un pamphlet : «La Vérité sur les États Confédérés d'Amérique " à Paris en $1862^{8}$. Pequet du Bellet travaille également avec Edward Gaulhiac, Créole comme lui, qui écrit exclusivement pour Le Constitutionnel de 
Paris - l'un des rares journaux favorables à la cause du Sud et dont les premiers articles comme ceux de du Bellet datent de mai 1861.

En effet, la première série d'articles de du Bellet est publiée dans Le Pays, où il défend l'esclavage $(8,13,20$, février 1861, traduite et publiée par le New York Herald en mars). Deux petits livres suivent : La révolution américaine dévoilée et Le blocus américain. En 1862, il publie deux autres monographies : Lettre à l'Empereur : De la reconnaissance des États Confédérés d'Amérique et Lettre sur la guerre américaine. Enfin, il publie en 1864 : l'Amérique du Nord. Lettre au corps législatif.

10 En 1865, après la guerre, il rédige ses notes sur les activités politiques des Confédérés en France. The Diplomacy of the Confederate Cabinet of Richmond and Its Agent Abroad est une critique sévère de l'inconsistance de l'action du gouvernement de Richmond et de ses représentants en Europe. Selon lui, le gouvernement du Sud, soutenu par une excellente armée et disposant de ressources importantes, est entré en guerre dans les meilleures conditions possibles. Pourtant, ce gouvernement a dilapidé le sang et les ressources de son peuple en accumulant faute sur faute. Du Bellet y relate les activités néfastes des plus hauts responsables confédérés à Paris qui, dans l'ensemble, ne trouvent aucune grâce à ses yeux : Pierre A. Rost, James Mason, John Slidell, Samuel Barron - officier en chef de la marine en Europe - Henry Hotze, et tous les autres responsables du ravitaillement en matériels et en fonds. Par ailleurs, il y glorifie sa propre intervention indispensable auprès de Français "bien placés", tel Émile Erlanger ${ }^{9}$ qui consent à prêter à la Confédération 5 millions de livres ou encore Louis Armand, membre du corps législatif, qui propose de construire des navires pour la Confédération. Après la guerre, désespéré par la défaite du Sud, il restera en France jusqu'à sa mort en 1884 .

11 Afin de contrecarrer la campagne sans précédent lancée dans la presse française par William Seward et William Dayton en faveur du Nord, du Bellet déclare, avec une certaine solennité, vouloir se battre en " prenant la plume ne pouvant prendre l'épée ». En effet, très rapidement, les journaux les plus vendus Le Siècle, l'Opinion Nationale, Le Temps, Les Débats, La Presse prennent fait et cause pour le Nord. C'est alors que Pecquet du Bellet prend l'initiative : en attendant l'arrivée de représentants officiels, il contacte Granier de Cossagnac, rédacteur en chef du Pays et réussit ainsi à publier une série d'articles. Comme il le clame à juste titre, il est encore considéré comme le représentant officiel du Sud, alors qu'il n'a aucun mandat. Son nom circule en effet dans les milieux de la presse car, contrairement à l'Angleterre, en France les articles sont obligatoirement signés et cependant, dans les journaux qui ouvrent leurs colonnes aux Confédérés, les journalistes français refusent d'endosser la responsabilité de propos favorables au Sud. Comme il le dit lui-même dans une boutade : « tout le monde pensait que j'étais l'envoyé de Davis jusqu'à ce que toute sorte de représentants militaires et financiers n'envahissent le Grand Hôtel » où "ils vivent et dépensent sans compter, passent leur temps à boire du champagne et à commenter les nouvelles alors que pour toute demande de financement des campagnes de propagande la réponse de Richmond est toujours non, faute d'argent » (Pequet du Bellet 1865, 51).

12 C'est notamment dans sa Lettre sur la Guerre Américaine - pamphlet publié à Paris en 1862 - qu'on retrouve exposés tous les arguments mis en avant à l'attention des Français. Cette lettre présente des arguments politiques, idéologiques et économiques.

13 L'argument politique comprend deux volets. Le premier est celui du droit à la séparation, du droit des États à rompre un contrat librement consenti. L'Europe, 
affirme-t-il, ne peut accepter l'enchaînement forcé des États qui ne le souhaitent pas alors qu'elle-même se modifie. Ce rappel du droit à la séparation étant exposé, du Bellet développe un autre aspect qui touche les Européens de plus près : celui des États-Unis comme "rouleau compresseur ». Ils se sont débarrassés de la "race indienne », ils feront bientôt de même de la "race noire " et finiront par effacer totalement la diversité européenne par l'absorption totale de l'Amérique centrale, du Mexique et du Canada. Ces individus dangereux sont, selon du Bellet, les gens du Nord incarnés par Lincoln qu'il présente comme une menace pour toute l'Europe. Cependant, à travers le mot "Europe ", c'est le mot "France " qu'il faut entendre, car si l'Angleterre ne reconnaît pas la Confédération, c'est parce qu'elle ne souhaite qu'une chose : la mort de la démocratie. Selon lui, en effet, l'Angleterre souhaite l'anéantissement de l'Amérique qui porte ombrage à son aristocratie : elle veut d'un même coup ruiner le Nord, rival maritime redoutable, ainsi que le Sud dont le coton fait concurrence à celui des Indes. La France, à ses yeux, reste donc la seule alliée du Sud.

Suit l'argument idéologique : le Nord est raciste et ne mène pas une guerre contre l'esclavage. En effet, dès le début de la guerre, Pecquet du Bellet prend rapidement conscience que le Nord tente en vain de défendre l'institution de l'esclavage. Il essaie de retourner la situation en réfutant l'argument nordiste selon lequel la cause de la guerre est l'abolition de l'esclavage. Il écrit à ce propos :

Ce mensonge politique a trop duré et causé trop de maux, il est grand temps de finir avec l'hypocrisie du Nord et le faux sentimentalisme à l'aide duquel ses amis n'ont que trop réussi à détourner les regards des masses de la véritable question qui se débat en Amérique [...] (Pequet du Bellet 1862, 7)

À l'instar de la Confédération du Sud, affirme-t-il, les États du Nord sont esclavagistes (Maryland, Kentucky, Missouri, Delaware, et Virginie Occidentale) et Lincoln ne mène pas cette guerre pour détruire l'Institution mais bien pour conserver le Sud dans l'Union, afin de recueillir pour le Nord le monopole de son travail.

Les deux parties sont esclavagistes, mais le Nord est raciste alors que le Sud ne l'est pas : « Le Sud ayant été contraint de recevoir, malgré lui, les Africains que l'Angleterre et les États-Unis jetaient sur son territoire, s'est efforcé d'adoucir leurs moeurs et les a habitués au travail et il continue son oeuvre de civilisation [...]» (Pequet du Bellet 1862, 8). À noter ici la référence aux États-Unis comme pays distinct. La patrie, c'est le Sud. Cette affirmation de l'appartenance au Sud et l'assimilation des États-Unis à une puissance étrangère se vérifient dans tous les pamphlets publiés à Paris.

Du Bellet conclut sur l'esclavage en s'interrogeant sur la partialité de la France et de l'Angleterre qui, tout en désapprouvant théoriquement cette pratique, acceptent l'institution lorsqu'il s'agit de l'Espagne, du Brésil, de la Turquie, de la Russie ou de la Hollande. Pourquoi donc cela serait-il un motif d'ostracisme politique lorsqu'il s'agit du Sud des États-Unis?

$\mathrm{Du}$ Bellet s'insurge enfin contre l'opinion publique qui ne mesure pas assez les conséquences économiques d'une défaite du Sud. Il s'adresse aux organes de presse, aux écrivains chargés d'éclairer les masses et les exhorte à changer d'attitude. Il leur reproche une prise de position plus passionnelle que réfléchie. Après avoir rappelé que l'on ne peut exiger du Sud qu'il fasse disparaître «d'un trait de plume une institution qui représente 14 milliards de francs » comme prix de sa reconnaissance, il insiste sur les conséquences désastreuses qui en découleraient sur « la race noire » qui serait alors ramenée à la barbarie, et sur l'Europe qui se trouverait de facto privée d'une énorme 
réserve de matières premières indispensables à son économie. Il cite en exemple et pour preuve la constante détérioration de la situation des ouvriers anglais. Mais, selon lui, l'Angleterre, cruelle envers ses ouvriers, ne réagira pas pour améliorer leur sort. Seule la France, soucieuse de son peuple, peut comprendre et doit intervenir en reconnaissant la Confédération.

19 Alors qu'il s'adresse à l'Europe, tout au long de son pamphlet, il distingue pourtant la France, humaine et humaniste, de l'Angleterre sans scrupule. Bien que jouant l'ambiguïté entre la fibre latine contre l'anglo-saxonnisme, son appel à la France transparaît à chaque ligne. Dans ses articles et lettres adressés aux journaux, il affirme d'ailleurs très souvent être Français de cœur, à l'exemple de tous les hommes du Sud : "Si nous plaçons notre main sur la poitrine des populations du Sud nous y sentons battre des cœurs français ${ }^{10} »$.

20 Cette affirmation des origines françaises et latines présentées comme l'essence même de ce Sud bafoué, nous allons la retrouver encore beaucoup plus fortement revendiquée par deux autres personnalités créoles : Charles Deléry, dans un style polémique et fougueux, puis Alfred Mercier, à travers une analyse géopolitique et stratégique pseudo-rationnelle et objective.

\section{Le Dr Charles Chauvin Boisclair Deléry}

21 À l'instar du Dr Mercier, le Dr Charles Deléry fait partie de ces nombreux médecins de la Nouvelle-Orléans à avoir épousé en parallèle une carrière littéraire et médicale. Ces praticiens se connaissaient et fréquentaient le même petit milieu de la bourgeoisie créole blanche. Enfants de planteurs, ils avaient, pour la plupart, fait leurs études à Paris, d'abord au lycée puis à la Faculté de médecine. Ils étaient restés ensuite environ un an comme interne des hôpitaux avant de revenir à la Nouvelle-Orléans pour y exercer. Pour ces jeunes Louisianais qui fréquentaient les milieux littéraires, Paris était la capitale des lettres et des arts.

Issu d'une famille créole de vieille souche, Charles Deléry est né en 1815 dans une plantation de la paroisse Saint-Charles. Comme Rouquette ou les frères Mercier, il est envoyé à Louis-le-Grand dès l'âge de 14 ans. En 1842, il obtient son doctorat de médecine et revient exercer à la Nouvelle-Orléans. Dès son retour, il se met à composer des poèmes et à écrire des essais. Il se targue d'avoir rencontré à Paris, Lamartine et Victor Hugo. Deléry s'intéresse également à la philosophie politique et suit de près les mutations politiques de la France. Il commente la Révolution de 1848 et auparavant, en 1847, il publie son Essai sur la liberté où il pose la question de l'intérêt individuel et général et définit ce qu'il entend par nativisme. Cet essai est très explicite sur sa conception de la patrie et de l'appartenance et l'on retrouvera ces mêmes arguments dans sa défense acharnée du Sud. Pour lui, la Patrie, c'est d'abord une langue et une terre :

L'on m'accusera de nativisme et je me garderai de m'en défendre : le nativisme bien entendu est un sentiment honnête, louable et commun à tous les hommes, excepté peut-être à ceux qui ont un intérêt direct à ne pas l'admettre. L'homme que l'exil ou le désir de s'instruire pousse loin de sa terre natale, verse parfois des larmes d'attendrissement à la vue d'un arbre qui lui rappelle sa patrie... À la révolution française pendant les massacres de septembre, un de ces hommes féroces qui arrachaient les victimes des prisons pour les conduire au tribunal de sang s'intéressa à un prisonnier parce qu'il parlait la Langue de son pays, voilà le nativisme pris en flagrant délit [...] (Deléry 1847, 15) 
Au début de la crise, Deléry, se prononce contre la Sécession. Il estime que le Sud n'est pas encore prêt. Mais quand la guerre éclate, en 1861, il épouse la cause confédérée sans plus d'états d'âme. Il est très actif et publie régulièrement dans L'Abeille. Très rapidement il met en avant l'héritage français de la Louisiane qui la distingue du Nord yankee. Même dans son hommage au Lieutenant-Colonel Charles C. Dreux, mort au champ d'honneur, la nostalgie de la France des Lumières est palpable : cette France au passé glorieux est en grand danger de disparition dans un monde en proie au déclin. Le 17 juillet 1861, il écrit dans L'Abeille :

Le Dernier Chant du Guerrier Orateur : Il était de ce monde où les plus belles choses ont le pire destin...

Je me rappelle avec attendrissement, le temps bien loin déjà, où vous ouvriez à Paris votre maison hospitalière à tous les Louisianais exilés. C'était le rendez-vous de tous vos jeunes compatriotes qui étaient allés au foyer des lumières pour y recevoir une éducation solide si hautement appréciée alors ; aujourd'hui de si peu de valeur dans notre pauvre pays dégénéré [...] (Deléry 1861)

En avril 1862, la Nouvelle-Orléans tombe aux mains de la flotte fédérale. Le major général B.F. Butler, symbole de la défaite et de l'humiliation, homme détesté des Louisianais, subit les foudres de la plume de Deléry qui exerce son talent de polémiste dans une série d'écrits contre lui. Le poème suivant, dédié à Butler, sera le poème de trop. Recherché par les hommes du général, il est contraint de quitter la Louisiane pour la France :

Tyran né de la plèbe, ô ! hideuse nature, $\mathrm{Au}$ gibet des coquins viens que je te mesure Quel ventre t'a voi ? Dans quel antre es-tu né ?

De quel nom t'appelr, ô monstre innominé?

Et comment n'as-t pas, reptile armé d'escailles, De ta mère, en nissant, déchiré les entrailles?

L'humanité rouit de honte et de colère

D'avoir à te cmpter comme homme, toi panthère,

Hyène, toi cacal, tigre tout à la fois,

Synthèse des fléaux relégués dans les bois.

Etc... etc... en veux-tu, en voilà. (Tinker 1928, 118)

Deléry arrive donc à Paris en 1864 où il publie : Confédérés et Fédéraux. Les Yankees, fondateurs de l'esclavage aux États-Unis et initiateurs du droit de sécession (1864). De retour à la Nouvelle-Orléans après la défaite, il continue ses publications et critique violemment la Reconstruction. En 1868, il publie Le Spectre noir ou le radicalisme aux États-Unis. En 1877 il fait paraître une comédie en un acte : L'Ecole du Peuple où il met en scène deux politiciens noirs : Caïus Caesar Antoine, éphémère Lieutenant-Gouverneur de Louisiane et Percy Shelley Pinchback, candidat malheureux à la même fonction. La représentation est interdite à la Nouvelle-Orléans. Deléry quitte alors la ville en 1878 pour s'installer à Bay Saint-Louis, sur les rives du Mississippi, où il demeurera jusqu'à sa mort, à 73 ans.

Son pamphlet, Confédérés et Fédéraux sera publié, selon lui, en réponse à une diatribe de quarante-deux pages d'un certain Eugène Pelletan, "publiciste» français. C'est L'Adresse au roi Coton, qui fait l'éloge des Yankees, de Lincoln et de John Brown. Deléry mène alors une défense très astucieuse de la cause du Sud et de l'esclavage. L'un de ses axes d'attaque, c'est d'opposer les Yankees, anglo-saxons, au Sud plus latin. Il commence par une charge en règle contre ces Yankees, dont les bateaux avaient amené les premiers esclaves d'Afrique, et dont les ancêtres de Nouvelle-Angleterre avaient 
détruit la tribu Pequot en 1637, après s'être partagés femmes et enfants et avoir vendu les hommes comme esclaves aux Indiens de l'Ouest. Il en déduit ainsi que « seuls les esclaves appartiennent au Sud, mais non l'esclavage » et conclut par cette boutade : "Quand on entend l'Angleterre gourmander le Sud au sujet de l'esclavage, il semble entendre une mère quereller sa fille à propos d'un vice de conformité dont elle l'aurait faite héritière ». Donc, l'origine de tous les maux du Sud vient des Anglo-Saxons incarnés par les Yankees du Nord.

Dans la foulée, et sur le même ton polémique, Deléry attaque l'Europe abolitionniste qui à ses yeux est d'une grande hypocrisie. Elle s'insurge contre l'esclavage en théorie et le favorise par son économie :

Les abolitionnistes européens n'ont pas réfléchi qu'il y avait, dans le principe, un moyen bien simple d'arriver à l'extinction de la servitude involontaire sans l'effusion d'une seule goutte de sang. C'eût été de ne pas établir chez eux de manufactures au service du roi coton ou de fermer plus tard quand leur sommeil s'est vu troublé par des scrupules de conscience. C'eût été à coup sûr, une mesure bien pacifique et bien efficace. On a découvert la conspiration du silence ; en voici une nouvelle, la conspiration de la consommation (Deléry 1864, 5).

Il est intéressant ici de donner un large extrait du deuxième axe d'attaque de Deléry, cette fois sous l'angle religieux : les Yankees anglo-saxons et puritains sont responsables de la guerre et de tous les maux dont souffre le Sud :

Ce qui est révoltant d'impudence et d'hypocrisie, c'est d'entendre les Puritains de la Nouvelle-Angleterre vociférer de leur voix nasillarde contre l'institution particulière, eux qui ont monopolisé le trafic de chair humaine, qui ont repoussé toute mesure tendant à l'abolition de la traite, et qui ont pieusement vendu leurs esclaves à ce même Sud, dans le but sans doute de soulager leurs consciences.

Selon divers besoins, il est une science

D'étendre les liens de notre conscience

et de rectifier le mal de l'action

Avec la pureté de nos intentions.

Ils n'étaient pas à leur coup d'essai quand ils se mirent à vendre les peaux noires ; ils avaient déjà fait le trafic des peaux rouges (Deléry 1864,6)

Il revient en détail sur l'histoire des Puritains, sur le caractère intolérant et sectaire de leur religion. Il cite les exemples de William Rogers et Ann Hutchinson, enchaîne avec les sorcières de Salem (1648) et conclut en déclarant qu'il faut admettre avec M. Cox de l'Ohio : «[...] que c'est la tendance des puritains à propager leur doctrine d'une manière offensive qui leur a valu d'être persécutés dans la mère patrie » (Deléry 1864, 13).

On perçoit alors le glissement opéré par Deléry d'une critique du puritanisme vers une critique du protestantisme en passant par le judaïsme. On retrouve là tous les ingrédients du rejet du protestant par le latin catholique :

Si une chose a lieu d'étonner, c'est de voir un peuple fuyant devant la persécution ériger, en arrivant dans sa nouvelle patrie, l'intolérance en système et se montrer mille fois plus rigoureux envers les dissidents que leurs adversaires ne l'avaient été à leur égard ; c'est de voir un peuple chrétien né d'hier, désertant le Christ pour Jéhovah, aller puiser ses inspirations sociales et législatives dans un livre tout frissonnant encore des colères et des malédictions du Dieu des Juifs.

Les Puritains y ont en effet, trouvé leur gibier, comme aurait dit Montaigne, c'est-à-dire la justification de leur nature intolérante et persécutrice. Il faut remonter jusqu'au $\mathrm{XVI}^{\mathrm{e}}$ siècle, à la sanglante époque du fougueux Luther et du féroce Calvin, pour trouver le pendant des horribles scènes de persécution dont les pèlerins de la Nouvelle-Angleterre se sont rendus coupables envers de prétendus hérétiques, comme si l'hérésie pouvait exister à l'égard du protestantisme, et 
particulièrement à l'égard d'une secte (les Puritains) qui rejette toute tradition, toute autorité humaine, et dont chaque membre, pour être conséquent au principe, devrait être son propre prêtre (Deléry 1864, 12).

31 Il conclut enfin en forme d'avertissement à la France : "C'est le même fond de fanatisme, la même roideur de caractère, le même orgueil qui lui fait s'adjuger aujourd'hui le titre de nation universelle, à l'exclusion de la vieille France qui épanche généreusement sur le monde ses lumières et la civilisation » (18).

Malgré un style totalement différent, on retrouve chez Deléry la même peur agitée contre le Nord par du Bellet : la capacité de ces Yankees à faire disparaître toute autre culture, surtout si elle est latine catholique et française. Ainsi, selon ces créoles, l'esclavage n'est plus au cœur de la guerre ; les enjeux sont autres : il en va de la survie de la race latine dans les Amériques.

Les enjeux géostratégiques et politiques exposés par Alfred Mercier viennent apporter une certaine légitimité et rationalité aux arguments offerts par Deléry, sur le mode très polémique, ou par du Bellet, de façon plus diplomatique.

3. Le Dr Alfred Mercier

34 Jean, le premier des Mercier qui arrive en Louisiane est né à Blanquefort (Gironde) en 1772. La Terreur de 1793 le contraint à émigrer. Il s'installe à la Nouvelle-Orléans, se marie avec une Canadienne, devient planteur, élève une famille nombreuse et meurt en 1841. Il laissera deux filles, dont l'une épousera Pierre Soulé, et trois fils : Jean-Jacques, Armand et Alfred. Alfred est né en 1816. À 14 ans, comme Deléry, il est envoyé au lycée Louis-le-Grand à Paris, où son frère aîné l'avait précédé. Il s'inscrit ensuite à la Faculté de droit de Paris. Attiré par les lettres, il suit quelques conférences à la Sorbonne. Il accompagne également son frère à la Faculté de médecine. En 1838, de retour à la Nouvelle-Orléans, il abandonne le droit, passe un an à Boston pour apprendre l'anglais qu'il maîtrise mal et, en 1842, retourne à Paris où il publie son premier livre, Poèmes Romantiques. Il entreprend alors un voyage à pied à travers l'Italie et, en 1873, publie le Fou de Palerme (Voyage en 1847). Il est présent à Paris lorsqu'éclate la Révolution de 1848. Correspondant de La Chronique, journal de la Nouvelle-Orléans, il rédige différents articles qui rendent compte de la conduite du peuple parisien et du spectacle qu'offre la capitale au cours de ces journées mémorables. En 1851, il assiste également au couronnement de Napoléon III. À 33 ans, il s'inscrit à la Faculté de médecine et obtient son diplôme en 1855. Il retourne alors à la Nouvelle-Orléans, où il exerce pendant trois ans. De retour Paris en 1859, il est encore là lorsqu'éclate la guerre de Sécession. Mercier va consacrer tous ses efforts à tenter d'amener la France vers une alliance avec la Confédération. Il publie une brochure qui laisse percevoir que la France est menacée par l'union anglo-saxonne formée par l'Angleterre et les États du Nord et suggère comme contrepoids une alliance entre la France, les Confédérés, le Mexique et les autres pays latins d'Amérique.

La guerre terminée, il rentre définitivement à la Nouvelle-Orléans où il exerce de nouveau la médecine. Il fonde l'Athénée louisianais pour la défense de la langue française, puis il publie en 1877 La fille du prêtre (contre le célibat des ecclésiastiques). En 1881, il fait paraître L'habitation Saint-Ybars dont Lafcadio Hearn fait l'éloge. Il sera le premier à publier, dans les comptes rendus de l'Athénée, les prémices de la première Grammaire du patois négro-français à la Louisiane. Nommé officier de la légion d'honneur en 1885, il meurt en 1894 en prédisant, dès 1891, la disparition de la langue française. 
36 Dans son pamphlet Du Panlatinisme : nécessité d'une alliance entre la France et la Confédération du Sud publié en 1862, Alfred Mercier, loin du ton polémique de Deléry, défend une stratégie politique et militaire basée sur une analyse géostratégique émanant du constat qu'il fait de la situation sur le continent américain.

Selon Mercier, trois forces ou éléments de civilisation, se répandent sur le monde et tendent à se partager l'avenir : le russoslavisme, l'anglosaxonisme et le gallo-latinisme.

On y retrouve le spectre de la race anglo-saxonne dominatrice, " rouleau compresseur » éliminant toutes les autres races, mais Mercier, contrairement à du Bellet, présente les Anglo-Saxons avec une certaine fascination pour leur sens du progrès. Selon lui, ils avancent et transforment l'état sauvage en civilisation. Cependant, ils sèment sur leur chemin la destruction et surtout ne tolèrent aucune autre « race ». Il affirme même que la « race noire » ne doit sa survie qu'à l'institution de l'esclavage et grâce à un peuple latin qui tolère sa proximité.

39 Cet extrait résume bien à la fois le rejet et la fascination que représente pour Mercier le Yankee anglo-saxon :

Tandis que l'Angleterre enseigne le commerce et la civilisation européenne aux innombrables peuples d'Asie, de l'Afrique et de l'Océanie, les descendants de ses fils, renforcés par les intarissables émigrations de l'Allemagne et de l'Irlande, suppriment les forêts d'Amazonie et des bords de l'océan Atlantique aux rivages de l'océan Pacifique, depuis l'État du Maine et le territoire de Washington jusqu'au Rio Grande, improvisent des villes et créent des peuples avec une rapidité qui n'a pas d'analogue dans l'histoire. Sur cet immense théâtre, l'élément anglo-saxon a effacé tous les autres [...] il est en voie d'absorber toutes les variétés de la race blanche. Quant aux races rouge et noire, il a détruit la première en grande partie ou en a violemment rejeté presque tous les restes aux extrêmes confins de sa sphère d'action ; et la seconde, au Nord, il l'a repoussée avec ce froid et dur orgueil de caste qui voit une souillure même dans un simple voisinage ; tandis qu'au Sud, il s'est juxtaposé à elle dans des conditions plus sociables de maître et d'esclave (Mercier 10)

Pour dramatiser encore plus la situation et provoquer des sentiments de compassion pour le Sud, il présente les Confédérés comme dernier rempart humain prêt à se sacrifier pour sauver le monde latin du dieu destructeur des Yankees. Avec beaucoup d'habileté, il rappelle à la France ses pertes déjà subies en Amérique latine et la situation préoccupante au Mexique. Il rappelle les humiliations passées en mentionnant au passage Napoléon à Saint-Hélène. Il conclut enfin sur la disparition de la langue et de la culture française de toutes les contrées conquises par les Anglo-Saxons. Mercier tente ainsi dans ce pamphlet de rallier l'opinion française à la cause du Sud en jouant invariablement sur la fierté et l'orgueil d'un peuple et en faisant apparaître son déclin partout à travers le monde. La France, sous-entend-il, est une puissance en perte de vitesse et en grand danger de disparition :

Les Confédérés (8 millions) ne se font pas d'illusion sur le sort qui les attend, s'ils sont vaincus ; ils savent jusqu'où peut aller le fanatisme des sectaires du Nord, fanatisme dont certainement on ne se fait pas une idée exacte en France, et qui est, à proprement parler, la monomanie ambitieuse et furieuse d'un peuple qui se croit véritablement l'élu du Dieu des armées, du Dieu exterminateur... Depuis quelques années, un mot encore vague circule dans le monde principalement sur les rivages de l'Amérique centrale et méridionale ; c'est celui de Panlatinisme.

41 Les populations d'Amérique d'origine dite latine s'inquiètent évidemment de l'expansion toujours croissante de la race anglo-américaine. L'instinct de conservation 
les porte à se demander si, en s'alliant entre elles, il ne leur était pas possible de s'opposer au flot envahisseur :

Quand le peuple envahisseur devient rapidement plus nombreux que le peuple envahi, qu'il substitue sa langue à la sienne, ses lois à ses lois, c'est l'envahi qui est absorbé par l'envahisseur. Veut-on obtenir une démonstration?

Que l'on considère ce que sont devenues les anciennes colonies françaises de l'Amérique septentrionale agrégées seulement depuis moins d'un demi-siècle à la grande famille Anglo-américaine ? Qu'en reste-t-il comme caractère primitif du Français, comme importance politique et commerciale ? Si peu que rien. Être de la même race et de la même religion, pour parler le langage de Napoléon à Ste-Hélène, cela fait partie de la destinée.

Si le Panlatinisme veut dire l'ambition naissante chez certains peuples (l'Italie, l'Espagne, et le Portugal) avec la France à leur tête, de disputer à la race anglo-saxonne la palme de l'initiative et du progrès, rien de mieux (Mercier 14-15).

Mercier développe l'idée que le Yankee, inspiré par la doctrine Monroe, est prêt à conquérir jusqu'au Cap Horn : « les Américains, dit-il en s'excluant totalement de cette appartenance, vont bientôt envahir le Canada sans opposition de l'Angleterre qui préfère le commerce avec les États-Unis. " Le Nord ne cherche donc pas à sauver l'Union mais l'unité du territoire en anéantissant les Sudistes. La finalité du Nord est bien de remplacer ces Latins par «les hordes barbares d'immigrants d'Angleterre de Suède, de Norvège ou d'Irlande » (Mercier 23).

Pour renforcer encore plus son argumentation d'un rêve hégémonique américain, Mercier cite la déclaration du gouverneur du Massachusetts, Pownall, datée de 1756 :

L'Amérique prend les proportions d'un État indépendant ; elle s'approche de ce but avec une rapidité dont l'Europe n'offrit jamais d'exemple. Elle deviendra l'arbitre du monde commerçant, et peut-être la dispensatrice de la paix générale. Elle usera de la force qu'elle sent en elle-même pour établir un système et changer celui de l'Europe (Mercier 26).

Alfred Mercier conclut en rappelant que la guerre n'est pas une simple affaire d'esclavage et d'émancipation. Selon lui, les « Américains » risquent d'établir un empire semblable à celui des Romains. Ils deviendront les régulateurs souverains du commerce maritime. Il faut donc ériger une digue pour s'opposer à cette expansion menaçante. Le Sud est prêt à jouer ce rôle mais la France, qui a accompli un acte de prévoyance en rendant la vie et la force au peuple mexicain, doit maintenant conclure une alliance avec les Confédérés pour compléter la tâche.

5 Il est vrai que cette idée qui s'est développée dans le Sud pour rallier la France n'a pas eu beaucoup d'impact, le factum de Mercier non plus. Mais l'intérêt de son pamphlet comme celui des deux autres, c'est de permettre de recouper les arguments développés par les créoles à Paris qui montrent une spécificité louisianaise, parfois en contradiction avec la propagande à l'intention de l'opinion publique anglaise.

Les trois créoles francophones que nous venons d'évoquer s'expriment en tant que Louisianais et Sudistes, soucieux de préserver la présence française sur le continent américain. Ils s'expriment et se reconnaissent comme Français d'Amérique et non comme Américains. On remarquera la condamnation des "Américains" à plusieurs reprises, surtout par Mercier et Deléry. Le puritanisme et l'anglo-saxonisme sont vertement critiqués alors que le catholicisme et le latinisme sont fortement mis en évidence. La doctrine Monroe comme principe « d'invasion » et de conquête sans limite est reprise par les trois pamphlétaires, et par bien d'autres encore qui n'ont pu être étudiés ici. 


\section{BIBLIOGRAPHIE}

Sources primaires

[Anonyme]. La Guerre Civile aux États-Unis. Impuissance du Nord, l'Indépendance du Sud inévitable, par A.D. Revenu Récemment des États-Unis. Paris, 1862.

Deléry, Charles. Essai sur la Liberté. Nouvelle-Orléans ,1847.

Deléry. Les Yankees fondateurs de l'esclavage aux États-Unis et Initiateurs du droit de Sécession. Paris, 1864.

Deléry. L'Abeille Louisianaise

Delelis, O. Leon. Scrapbook, 1843-1874. MSS. 2677, 2677-88. Hill Memorial Library. Louisiana State University, Baton Rouge, Louisiana. UU : 252.

Dumez, Eugène. Correspondance Dumez-Vignaud. 1862-1879. Vignaud Henry Papers. Louisiana Collection of LSU Libraries Special Collections [C-66, Box 1, folder 5,6 ${ }^{11}$.

Mercier, Alfred. Du Panlatinisme, Nécessité d'une alliance entre la France et la Confédération du Sud. Paris, nd.

Transatlantica, 1 | 2002 
Musson, Eugène. Lettre à Napoléon III sur l'esclavage dans les États du Sud par un Créole de la Louisiane. Paris, 1862.

Pecquet du Bellet, Paul. Lettre sur la Guerre américaine. Journal Le Pays. Paris, 1862.

Pecquet du Bellet, Paul. « The Diplomacy of the Confederate of Richmond and its Agents Abroad : Being Memorandum Notes Taken in Paris during the Rebellion of the Southern States from 1861 to 1865 ». Manuscrit publié. Confederate Centenarial Studies, 1963.

Sources secondaires

Beckles, Wilson. John Slidell and the Confederate in Paris: 1862-1865. New York : Milton, Balsh \& Co, 1932.

Bullock, James. D. Secret Service of the Confederate States in Europe: Or How the Confederate Cruisers Were Equipped. New York : Modern Library War, 2001.

Cullop, Charles. Confederate Propaganda in Europe : 1861-1865. Coral Gables,Florida : University of Miami Press, 1969.

Hall, Gwendolyn Mildo. Africans in Colonial Louisiana. Baton Rouge : Louisiana State University Press, 1992.

Tinker, Edward, Laroque. Les Écrits de Langue française en Louisiane. Paris : H. Champion, 1928.

Tinker, Edward, Laroque. French Newspapers and Periodicals of Louisiana. Worcester, MA : American Antiquarian Society, 1933.

Viatte, Auguste. « Complément à la bibliographie louisianaise d'Edward Laroque Tinker ». Revue de Louisiane III (1974) : 13-57.

\section{NOTES}

1. Après l'achat de la Louisiane par les États-Unis, les Louisianais d'origine française et espagnole se présentent comme créoles pour se distinguer des Anglos, mais c'est surtout après la guerre de Sécession qu'ils s'approprient le terme alors que celui-ci continue à être revendiqué par les gens de couleur libres. J'adopterai la définition de Gwendolyn Mildo Hall pour qui est créole toute personne de parents non américains nés dans les Amériques. Pour des raisons de clarté, on distinguera les Créoles de couleur des Créoles blancs, ces derniers faisant l'objet de la présente analyse (Hall 263).

2. Le XIX ${ }^{e}$ siècle voit l'explosion de la presse en langue française. Entre 1840 et 1850, on dénombre 33 journaux à la Nouvelle-Orléans et 17 dans les paroisses. Cette vitalité est, en partie, due aux vagues successives de Français qui s'y réfugient : réfugiés de la Monarchie de Juillet, de la révolution de 1848 et enfin du second Empire.

3. Malheureusement, le Mexique qui incarne le plus important effort français accompli outre-mer en dehors de l'Algérie sous le Second Empire sera aussi le plus grave échec. Après la guerre de Sécession, les Américains signifient clairement à Napoléon qu'ils comptent bien appliquer la doctrine Monroe, ce dernier relève Maximilien de ses fonctions sous prétexte d'une mauvaise gestion, et, lorsque l'archiduc refuse d'abdiquer, il est fusillé en 1867.

4. Les pamphlets analysés ne constituent que quelques exemples parmi les nombreuses publications en faveur du Sud rédigés par les Créoles à Paris. 
5. Un exemple de cette double allégeance est la large diffusion du Chant de Guerre du Sud (5 strophes) sur l'air de la Marseillaise publié en juillet 1861 (Leon Delelis Papers) et dont voici la seconde strophe pour illustrer :

Quittons nos toits, notre famille,

Les champs féconds qu'aiment nos cœurs,

Et jurons sur ce fer qui brille,

Jurons de revenir, vainqueurs! (Bis)

Partons l'âme fière et ravie ;

Marchons en avant, l'arme au poing ;

Femmes sur nous ne pleurez point ;

Notre sort est digne d'envie!

Au nom de la patrie, armons nous citoyens !

Luttons (Bis) pour maintenir nos libertés, nos biens !

6. Il existe deux versions manuscrites du mémorandum dans les archives de Pequet du Bellet.

7. Une copie dactylographiée du manuscrit est parvenue à la Library of Congress en 1902, envoyée par un certain M.T. Turner de Paris. C'est cette version qui fait l'objet d'une publication en 1963. L'éditeur reconnaît ne pas être en mesure de localiser la version manuscrite. En 1998, Nicolas Kariouk, descendant de Pecquet du Bellet, a fait don à la Hill Memorial Library, de certaines archives de la famille dans lesquelles se trouve la version manuscrite.

8. Edwin de Leon est très actif. Il dépense des sommes importantes en pots-de-vin pour rallier à la cause du Sud un certain nombre de journalistes. Il est méprisé et ignoré par John Slidell représentant officiel de la Confédération à Paris. L'occasion se présente à Slidell de se débarrasser de de Leon lorsqu'une lettre adressée par ce dernier à la Confédération est interceptée par le Nord et publiée. Dans cette lettre, de Leon critiquait Slidell et la presse française. Il est limogé au début de 1864.

9. Emile Erlanger, banquier influent, lance le « cotton loan " pour venir en aide au Sud. Il consent à la confédération un prêt de 5 millions de livres et épouse la cause en même temps que Matilda, la fille cadette de John Slidell.

10. Affirmation parue dans une lettre au journal Le Pays en juin 1861 à laquelle répond le 20 juin un certain Adam Gurowski, citoyen des États-Unis, accusant les Confédérés d'opportunisme. Leur cœur est français à Paris et anglais dans le London Times.

11. De 1862 à 1864, il écrit de France, puis retourne à la paroisse Saint Jean-Baptiste où il continue sa correspondance avec Vignaud, resté lui à Paris.

\section{RÉSUMÉS}

During the Civil war, the French-educated Creole elite of Louisiana played an important part in efforts to persuade France to support the Confederacy. This article analyses the arguments put forward by three famous Creoles living in Paris during the Civil War (Paul Pequet du Bellet, Dr Alfred Mercier and Dr Charles Delèry) to rally French public opinion to the cause of the Confederacy. Faced with a strong anti-slavery sentiment, they argued that France's interest lies in defending the South and its « Latin race » from the aggressions of the Anglo-Saxon North. 
INDEX

Mots-clés : Louisiane, Guerre de Sécession, Confédérés, Etats-Unis

Keywords : créole, Louisiana, Civil War, creole, United States, Confederacy

\section{AUTEUR}

\section{SALWA NACOUZI}

Salwa Nacouzi est maître de conférences à l'Université de Poitiers. 